\title{
PHOTOMETRIC DATA IN MACHINE-READABLE FORM
}

\author{
B. HAUCK
}

Institut d'Astronomie de l'Université de Lausanne et Observatoire de Genève

\begin{abstract}
For two years we have undertaken the collection of photoelectric data in view of their utilization by computer techniques. At present we are preparing a fine for each photometric system with the measurements published. As a second step, we shall form another file, alike for each system, with homogeneous measurements. This file will be obtained with weighed means of the published measurements. For that we need to compare each published list with a standard list before attributing the weights.

After that, we shall form a file indicating in which system a star is measured.

This work is undertaken in connection with the Data Center of Strasbourg and the group on spectroscopic and photometric data of the Commission 45.
\end{abstract}

\section{Introduction}

For the last two years we have been collecting photometric photoelectric measurements. It is very important to render this information available to processing by computer techniques. At the present we are setting up files for each photometric system. The second step of this work will be the establishing of a file with homogeneous data for each system. Finally, a last file will permit us to know in which systems a star has been measured.

This work is included in the study undertaken by the Stellar Data Center of Strasbourg (Jung, 1971).

On the other hand the constitution of the group on spectroscopic and photometric data of Commission 45 should allow us to bring the files up to date.

\section{Homogenization of Data}

The homogenization of data is the most delicate part of this work. It would certainly be an advantage to find homogeneous data in the literature! But this is not the case, and users of photometric data who are not specialists in this field can have difficulty in choosing the best value, somewhat like the photometrist who may find it difficult to choose the best spectral type.

The sources of inhomogeneity are numerous. However, the principal ones are:

(1) the photomultiplier;

(2) the filters;

(3) the methods of reduction outside the atmosphere;

(4) the lack, or the bad use of standard stars.

It would be dangerous to average all measurements concerning one star without examining the quality of each published list. To make this critical study, we establish, for each system, a publication or a set of publications, as reference. Then we go through the stars of the list to be studied and include those which are common to the reference. 
This comparison is made color by color, or parameter by parameter. A color 'reference' vs color 'list' diagram is constructed by the computer and the regression line is calculated by the method of least squares.

If the connection with the reference is correct, then the slope of this line is 1 and the $y$ intercept is zero. The standard error obtained is a quality index of the list.

In order to detect systematical errors, we then construct the diagram of the differences between the reference and the list for each color (or parameter) as function of the temperature parameter defined in the studied system.

With these comparisons it is possible to give a weight to the list.

Sometimes, the discrepancy will be too great and the list will not be included in the file. When this is the case the Data Center has to draw the user's attention to these discrepancies.

\section{Common File}

This file must enable one to know quickly in which systems a star is measured and to select thus the files of these systems (file with published data or weighted data).

With these three files we have the advantage of obtaining data without delay and loss of information since the published measurements are preserved. A second advantage is the existence of the mean values obtained after critical examination.

\section{Identification of Stars}

The identification of stars is a complex problem since a star can be identified in many ways. It is necessary to choose a means of identification allowing computer treatment and valid for all stars. For this point we shall adopt the same process as the Stellar Data Center of Strasbourg; namely the use of equatorial coordinates $\alpha, \delta$. It has been decided to use a code made with these two coordinates for the 1950,0 epoch.

\section{Comparison of Photometric Systems}

When all the information concerning a system has been collected, it will be possible to compare it with others. The theoretical study of the comparison of two systems has been made by Golay (1959). For the comparison of the astrophysical information contained in two different systems, it is necessary to take some precautions. In particular, one must define for what types of stars a relation between two indices (or parameters) is valid. Then one must examine the validity of this relation for different samples, for example Am, subdwarfs, giants, supergiants, stars with interstellar reddening, etc. (Hauck, 1971). This work has been rarely done in the past.

\section{State of Work at Present}

We have established files 1 and 2 for the following systems: (1) six-colors of Stebbins and Whitford; (2) uvby $\beta$ ( 5000 stars). 
Some systems can be considered as homogeneous, because all data come from the same observation group. They are the rather well-known systems by: (1) Borgman; (2) Kruszewski; (3) Argue; (4) Geneva Observatory; (5) Eggen (10200, $6500 \AA$, $6200 \AA$ ); (6) Vilnius Observatory; (7) Tifft; (8) Bahng.

For the $U B V$ system we have continued the work of Blanco et al. (1970), especially for the galactic star clusters (100 lists and 13000 measurements).

\section{References}

Blanco, V. M., Demers, S., Douglass, G. G., and Fitzgerald, P. M.: 1970, U.S. Naval Obs. Publ., 2nd Ser. 21.

Golay, M.: 1959, Publ. Obs. Genève Série A, No. 60.

Hauck, B.: 1971, Conf. Oss. Astron. Milano-Merate Serie I, No. 12.

Jung, J.: 1971, Inform. Bull. CDS Strasbourg, Nos. 1 and 2. 